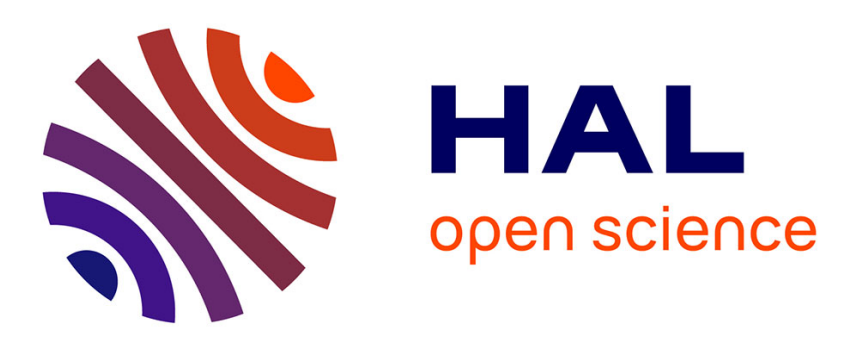

\title{
Interval analysis on non-linear monotonic systems as an efficient tool to optimise fresh food packaging
}

\author{
Sébastien Destercke, Valérie Guillard
}

\section{To cite this version:}

Sébastien Destercke, Valérie Guillard. Interval analysis on non-linear monotonic systems as an efficient tool to optimise fresh food packaging. Computers and Electronics in Agriculture, 2011, 79 (2), pp.116124. 10.1016/j.compag.2011.08.014 . hal-00655322

\section{HAL Id: hal-00655322 \\ https://hal.science/hal-00655322}

Submitted on 27 Dec 2011

HAL is a multi-disciplinary open access archive for the deposit and dissemination of scientific research documents, whether they are published or not. The documents may come from teaching and research institutions in France or abroad, or from public or private research centers.
L'archive ouverte pluridisciplinaire HAL, est destinée au dépôt et à la diffusion de documents scientifiques de niveau recherche, publiés ou non, émanant des établissements d'enseignement et de recherche français ou étrangers, des laboratoires publics ou privés. 


\title{
Interval analysis on non-linear monotonic systems as an efficient tool to optimise fresh food packaging.
}

\author{
Sebastien Destercke ${ }^{\mathrm{a}, \mathrm{b}, *}$, Valerie Guillard ${ }^{\mathrm{c}}$ \\ aINRA/CIRAD, UMR1208, 2 place P. Viala, F-34060 Montpellier cedex 1, France \\ ${ }^{b}$ LIRMM, UMR 5506, 161 rue Ada, 34095 Montpellier \\ ${ }^{c}$ Université Montpellier 2, UMR1208, 2 place P. Viala, F-34060 Montpellier cedex 1, France
}

\begin{abstract}
When few data or information are available, the validity of studies performing uncertainty analysis or robust design optimisation (i.e., parameter optimisation under uncertainty) with a probabilistic approach is questionable. This is particularly true in some agronomical fields, where parameter and variable uncertainties are often quantified by a handful of measurements or by expert opinions. In this paper, we propose a simple alternative approach based on interval analysis, which avoids the pitfalls of a classical probabilistic approach. We propose simple methods to achieve uncertainty propagation, parameter optimisation and sensitivity analysis in cases where the model satisfies some monotonic properties. As a real-world case study, we interest ourselves to the application developed in our laboratory that has motivated the present work, that is the design of sustainable food packaging preserving fresh fruits and vegetables as long as possible.
\end{abstract}

Keywords: Interval analysis, robust design, fuzzy sets, sensitivity analysis, uncertainty analysis.

\section{Introduction}

There are many sources of uncertainties in life science and in agronomy, the main reasons for it being the high variability of living organism and the error of measurement devices. Also, the number of available samples for a given experiment may be limited (sometimes even reduced to one sample), due to cost or

\footnotetext{
${ }^{*}$ Corresponding author

Email addresses: sebastien.destercke@cirad.fr(Sebastien Destercke), guillard@univ-montp2.fr(Valerie Guillard )
} 
practical limitations. In such situations, it can be hard to determine a meaningful probabilistic model of the parameters, let alone a joint probabilistic model over all parameters. In other situations, uncertainty around a parameter or a constant can be described by expert opinions, and whether these opinions can be faithfully translated by single probabilities is questionable (see, e.g., [28, Sec. 4] or [26]).

In such situations, it may be better to use interval modelling and interval analysis to perform uncertainty studies, simply because determining intervals requires less data and knowledge. Also, using interval analysis amounts to make no assumptions about parameter dependencies.

In this paper, we consider dynamical non-linear models describing the evolution of variables, with the aim to optimise some of the parameter values w.r.t. some given objective. In such systems, the values of initial conditions, non-modifiable parameter values or even of the objectives may be ill-known. It is then desirable to perform some uncertainty analysis to achieve robust design. Performing such analysis with classical probabilistic methods $[15,4]$ usually requires to:

- specify the distribution of each input variable,

- specify the dependency structures between input variables,

- perform (costly) numerical analysis to evaluate the output uncertainty.

Meeting such requirements necessitates an important amount of information and data. It also involves the use of techniques having a high computational cost. In practice, when not enough information is available, distribution shapes (e.g., normality) and dependence assumptions (e.g., independence between all variables) are often chosen accordingly to some practical criterion rather than to available information. However, the validity of such choices, not confirmed by experiments or available knowledge, may be questioned, as well as the validity of subsequent analysis results [9]. They then provide overly precise and misleading conclusions, which may in turn lead to unwarranted and non-robust design choices.

When few data are available, an alternative is to use interval analysis [16] to perform the uncertainty analysis, that is to consider that only the bounds in which each parameter may vary are known (an information that is often available). Such an analysis comes down to consider that:

- variable and parameter distributions are unknown (up to their bounds),

- dependence structure between variables and parameters is unknown. 
Compared to probabilistic analysis, interval analysis can therefore be seen as a conservative analysis, in the sense that it does not make any additional hypothesis with regard to the available information, and possibly ignores some of the available information. However, in scientific analysis as well as in robust design, it is safer to use such conservative methods than to make unsupported assumptions. Also note that, when function $f$ has some monotonic properties $[27,11]$ (a common case in life sciences and other domains [24,3], where simple models are often encountered), performing interval analysis may require very few computations compared to, say, probabilistic Monte-Carlo analysis. When these monotonic properties are not satisfied, performing interval analysis requires more complex techniques [23] with increased computational complexity (the computational cost then becoming comparable to the one of probabilistic methods). However, whether the monotonic properties are satisfied have no impact on the results conservativeness of interval analysis.

In this paper, we introduce a set of methods to achieve uncertainty propagation, parameter optimisation and sensitivity analysis on monotonic dynamical systems when uncertainty is described by intervals. Notations and general problem formulations are introduced in Section 2. Section 3 then provides details about the method themselves.

Finally, we illustrate the method on the real-world case study that have motivated the present work and that is currently treated in our laboratory [5] (see website http://www.tailorpack.com/). It concerns the design of sustainable fresh food packaging, with the objective to preserve food from decay as long as possible. We use our method to, first, perform uncertainty analysis of a model describing gas exchanges between the packaging atmosphere and exterior atmosphere and, second, optimise oxygen and carbon dioxide permeances of packaging materials for a given fruit or vegetable, here chicory. The whole case study is described in Section 4.

\section{Problem setting and notations}

Vectors of model variables and parameters will be denoted by bold letters $(\mathbf{x}, \mathbf{p}, \ldots)$, while specific values of these vectors will de denoted by non-bold letters $(x, p, \ldots)$. As the models considered in this paper are multivariate, indexed letters $\mathbf{x}_{j}$ will denote the $j$ th element of vector $\mathbf{x}$. Sets will be denoted by calligraphic letters $(\mathscr{X}, \mathscr{P}, \ldots)$. The real line will be denoted by $\mathbb{R}$.

We consider a dynamical non-linear model $\dot{\mathbf{x}}=f\left(\mathbf{x}, \mathbf{p}_{E}, \mathbf{p}_{D}, t\right)$ with $f: \mathbb{R}^{m+n+1} \rightarrow$

$\mathbb{R}^{m}$ a time-dependent function that describes the evolution of $m$ state variables 
$\mathbf{x} \in \mathbb{R}^{m}$, i.e. $\mathbf{x}=\left(x_{1}, \ldots, x_{m}\right)$. The evolution of these variables depends on $n_{E}$ environmental parameters $\mathbf{p}_{E}$ (whose values cannot be controlled) such as temperature or external pressure and $n_{D}$ design parameters $\mathbf{p}_{D}$ (which values can be modified) such as mechanical or chemical properties of synthetic compounds. They form a vector $\mathbf{p}=\left(\mathbf{p}_{D}, \mathbf{p}_{E}\right)$ of $n$ parameters $\mathbf{p}=\left\{p_{1}, \ldots, p_{n}\right\} \in \mathbb{R}^{n}$.

Given some values $p \in \mathbb{R}^{n}$ of the parameters and some initial conditions $x(0) \in$ $\mathbb{R}^{m}$ of the state variables, the solution of the system represented by $f$ at time $t$ is $x(t)=\left(x_{1}(t), \ldots, x_{m}(t)\right)$ where $x_{i}(t)$ describes the state of the $i$ th state variable $\mathbf{x}_{i}$ at time $t$.

When initial conditions $x(0)$ and environmental parameter values $p_{E}$ are known, a classical design problem [1] consists in identifying the values $\widehat{p}_{D} \in \mathbb{R}^{n_{D}}$ of the design parameters $\mathbf{p}_{D}$ so that the solutions $x(t)$ are as close as possible to a given objective $\widehat{x}(t)$ on the state variables. However, both the exact values of initial conditions $x(0)$, of parameters $p$ or of the objective $\widehat{x}(t)$ to reach are seldom known with certainty. In the next section, we detail how the problem can be treated when these values become interval and when the model satisfies some monotonic properties.

\section{Interval analysis and design optimisation}

In this section, we start by giving some refreshers on classical interval analysis and interval analysis on dynamical monotonic models, illustrating them on a simple example. We then detail our proposed optimisation and sensitivity analysis methods for such models.

\subsection{Basics of interval analysis}

In the computational literature, interval analysis was first developed to take account of numerical errors [19]. However, interval analysis is now mostly used to perform robustness analysis in applications (robotics [17], chemical, biological, ...) where variable values are imprecisely known [16].

A real interval $[x]:=\left[x^{-}, x^{+}\right]$of a variable $X$ is a connected and closed subset of $\mathbb{R}$. The set of real intervals is usually denoted $\mathbb{I R}$. An interval vector $[\mathbf{x}]$ over $\mathbb{R}^{n}$ (also called box) is the Cartesian product of $n$ intervals. The classical problem of interval analysis consists in replacing, in a given function $\mathbf{Y}=f\left(X_{1}, \ldots, X_{n}\right)$ from $\mathbb{R}^{n}$ to $\mathbb{R}^{m}$, the point values $\mathbf{x}=\left(x_{1}, \ldots, x_{n}\right)$ of variables $\mathbf{X}=\left\{X_{1}, \ldots, X_{n}\right\}$ by intervals $[\mathbf{x}]=\left(\left[x_{1}\right], \ldots,\left[x_{n}\right]\right)$ and to compute the range

$$
f([\mathbf{x}])=\{f(\mathbf{x}) \mid \mathbf{x} \in[\mathbf{x}]\} .
$$




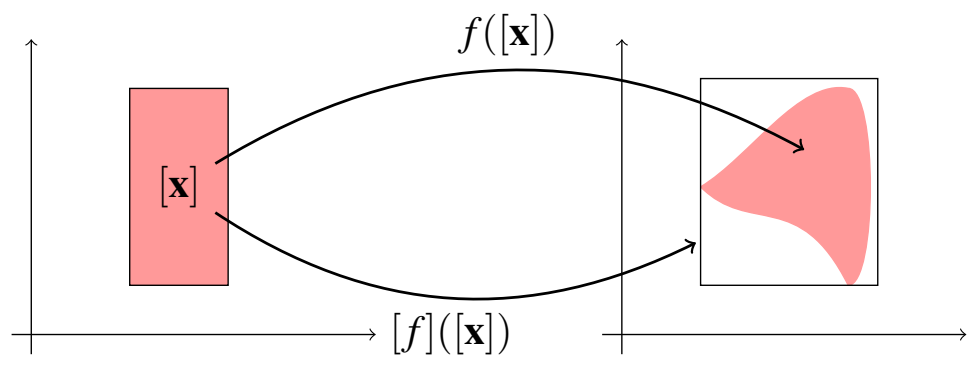

Figure 1: Illustration of interval analysis with inclusion function (Equation (2)).

Usually, $f([\mathbf{x}])$ is not a box over $\mathbb{R}^{m}$, but a complicated subset of it (i.e., it cannot be expressed as a Cartesian product of intervals). Rather than computing the exact propagation $f([\mathbf{x}])$, one can use guaranteed approximation techniques and compute a box $[f]([\mathbf{x}])$ over $\mathbb{R}^{m}$ that will be an inclusion function, i.e.,

$$
f([\mathbf{x}]) \subseteq[f]([\mathbf{x}]) .
$$

This comes down to compute an outer approximating interval for every dimension of $f$. The notion is illustrated in Figure 1. Among possible techniques to compute such inclusion functions is interval arithmetics [19], where classical arithmetic operations $\{+, \times, /,-\}$ are replaced by their interval equivalent. Depending on the technique and on the characteristic of the model $f,[f]([\mathbf{x}])$ will be more or less close to $f([\mathbf{x}])$. In the next section, we recall such techniques that can be applied to the particular dynamical models we are interested in.

\subsection{Interval analysis to propagate uncertainties in dynamical systems}

Let us consider the more complex problem of evaluating the solution of the dynamical system $\dot{\mathbf{X}}=f\left(\mathbf{X}, \mathbf{P}_{E}, \mathbf{P}_{D}, t\right)$ with $f: \mathbb{R}^{m} \rightarrow \mathbb{R}^{m}$. In this model, the evolution of each state variable $X_{i}, i=1, \ldots, m$ is described by an ordinary differential equation (ODE) such that $\dot{X}_{i}=f_{i}\left(\mathbf{X}, \mathbf{P}_{E}, \mathbf{P}_{D}, t\right)$. The solutions of such systems are $m$ functions $x_{i}(t), i=1, \ldots, m$. Here values of function $x_{i}(t)$ are discretised and computed for a finite number of time steps. We denote by $\mathscr{T}=[0, \bar{t}]$ the time domain of the model and assume that each $x_{i}(t)$ is computed for $T$ different times values $t_{k}, k=1, \ldots, T$ (with $t_{k}-t_{k-1}$ being constant for every $k$ ).

For such models, the classical problem of interval analysis is formulated as follows: given $m$ initial conditions $x_{i}(0) \in\left[x_{i}\right](0):=\left[x_{i}{ }^{-}, x_{i}{ }^{+}\right](0), i=1, \ldots, m$ and $n$ parameters intervals $p_{j} \in\left[p_{j}\right], j=1, \ldots, n$, find the bounds $[\mathbf{x}](t)=\left[\mathbf{x}^{-}, \mathbf{x}^{+}\right](t)$ 
of variable evolutions for each discretised time step $t=t_{k}, k=1, \ldots, T$. That is, determine the lower $\left(\mathbf{x}^{-}(t)\right)$ and upper $\left(\mathbf{x}^{+}(t)\right)$ envelopes of $\mathbf{x}(t)$ for each time step.

Let us call configuration an element of the Cartesian product $\mathscr{X}:=\times_{i=1}^{m}\left[x_{i}\right](0) \times{ }_{j=1}^{n}$ $\left[p_{j}\right]$. Interval analysis on $\dot{\mathbf{X}}=f\left(\mathbf{X}, \mathbf{P}_{E}, \mathbf{P}_{D}, t\right)$ then consists in finding, among all configurations in $\mathscr{X}$, those reaching the bounds of $\mathbf{x}\left(t_{k}\right)$ for each time step $t_{k}$, $k=1, \ldots, T$. As in the non-dynamical case (see Equation (2) and Figure 1), $[\mathbf{x}](t)$ can have a very complex shape, hence a slightly easier problem is to find, for each variable $X_{i}, i=1, \ldots, m$, some bounds $\left[x_{i}\right](t)$ such that $[\mathbf{x}](t) \subseteq \times_{i=1}^{m}\left[x_{i}\right](t)$ and then to consider the resulting boxes $\times_{i=1}^{m}\left[x_{i}\right](t)$ as the solutions. This is the problem we will consider here.

Among the set $\mathscr{X}$ of configurations, denote by $\mathscr{H}=\times_{i=1}^{n}\left\{x_{i}{ }^{-}, x_{i}{ }^{+}\right\} \times_{j=1}^{m}$ $\left\{p_{j}^{-}, p_{j}{ }^{+}\right\}$the set of extreme ones, that is the set of configurations whose elements are bounds of the intervals. $\mathscr{H}$ therefore contains only combinations of such bounds, i.e., $2^{(n+m)}$ elements, while $\mathscr{X}$ contains an infinity of them. To illustrate the approach used in this paper, we will use the following simple example:

Example 1. Consider the very simple system from $\mathbb{R} \rightarrow \mathbb{R}$

$$
\dot{X}_{1}=f_{1}\left(P_{1}\right)=P_{1},
$$

where $p_{1} \in\left[p_{1}\right]$ and $x_{1}(0) \in\left[x_{1}\right](0)$ are positive numbers, and the (analytical) solution of the system is $x_{1}(t)=P_{1} \cdot t+x_{1}(0)$. Here, both $\mathbf{X}=X_{1}$ and $\mathbf{P}=P_{1}$ are reduced to a single element each. Our knowledge about $p_{1}$ and initial condition is given by the intervals $p_{1} \in[0.5,1]$ and $x_{1}(0) \in[1,3]$. The sets of all possible combinations of $\left[p_{1}\right]$ and $\left[x_{1}\right](0)$, together with their extreme combinations are illustrated in Figure 2. In this example, the set $\mathscr{X}=[1,3] \times[0.5,1]$, while the set $\mathscr{H}=\{(1,0.5),(1,1),(3,0.5),(3,1)\}$ is reduced to four points.

As for interval analysis on classical functions [11], there exist specific cases [6, $24,27]$ where the bounds of $\left[x_{i}\right](t)$ on each dimension correspond to solutions of the system $\dot{\mathbf{X}}=f\left(\mathbf{X}, \mathbf{P}_{E}, \mathbf{P}_{D}, t\right)$ corresponding to specific points in the set $\mathscr{H}$ of extreme configurations, thus reducing the number of necessary computations to find them. Before recalling what are these cases, let us first define the notion of monotonic for dynamical system $\dot{\mathbf{X}}=f\left(\mathbf{X}, \mathbf{P}_{E}, \mathbf{P}_{D}, t\right)$.

Definition 1. $f_{i}$ is said to be dynamically increasing w.r.t. variable $X_{j}, j \neq i$ or parameter $P_{k}$ if

$$
\frac{\partial f_{i}}{\partial X_{j}} \geq 0 \text { or } \frac{\partial f_{i}}{\partial P_{k}} \geq 0
$$




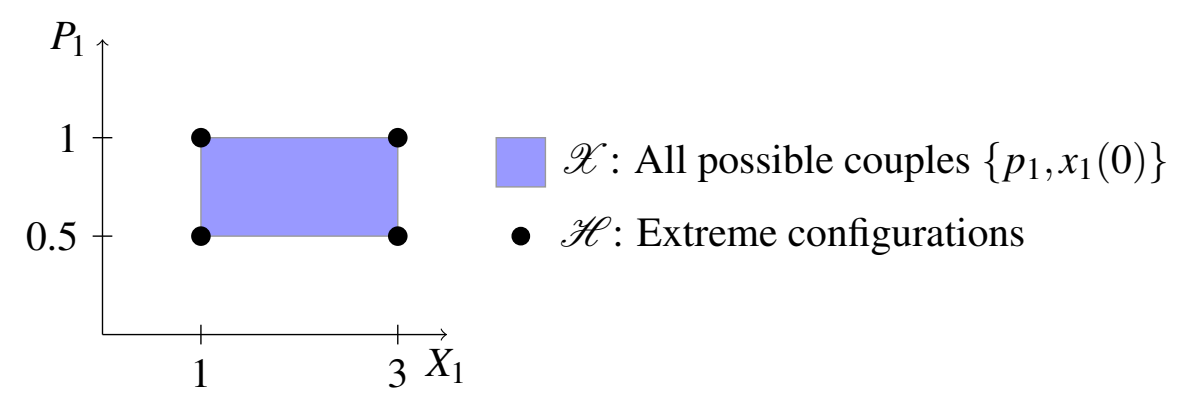

Figure 2: Example 1 set of (extreme) configurations.

and it said to be dynamically decreasing w.r.t. variable $X_{j}, j \neq i$ or parameter $P_{k}$ if

$$
\frac{\partial f_{i}}{\partial X_{j}} \leq 0 \text { or } \frac{\partial f_{i}}{\partial P_{k}} \leq 0
$$

For a given function $f_{i}$ (or, equivalently, for a given variable $X_{i}$ ), let us denote by $\mathbf{P}^{\nearrow, i}, \mathbf{X}^{\nearrow, i}$ and $\mathbf{P}^{\searrow}, i, \mathbf{X}^{\searrow, i}$ the set of parameters for which $f_{i}$ is dynamically increasing and decreasing, respectively. Note that they are disjoint, i.e. $\mathbf{P}^{\nearrow, i} \cap$ $\mathbf{P} \searrow, i=\emptyset$ and $\mathbf{X}^{\nearrow, i} \cap \mathbf{X}^{\searrow, i}=\emptyset$. We also assume from now on that these sets form partitions of the parameters and variables, that is for a given $i, \mathbf{P}^{\nearrow}, i \cup \mathbf{P} \searrow, i=\mathbf{P}$ and $\mathbf{X}^{\nearrow, i} \cup \mathbf{X} \searrow, i=\mathbf{X}$. When a function $f_{i}$ is dynamically increasing or decreasing in each variable and parameter, the following proposition [24] tells us how the upper and lower envelope of $\left[x_{i}\right](t)$ at any time step $t$ can be obtained.

Proposition 1. For any $t$, the bounds of $\left[x_{i}\right](t)$ can be computed as follows:

- $x_{i}^{+}(t)$ is the solution of the system $\dot{\mathbf{X}}=f\left(\mathbf{X}, \mathbf{P}_{E}, \mathbf{P}_{D}, t\right)$ with the configuration $\overline{\mathscr{H}}^{i} \in \mathscr{H}$ such that

$$
\overline{\mathscr{H}}^{i}=\left\{\begin{array}{cccc}
P=p^{+} & \text {for all } & P \in \mathbf{P}^{\nearrow, i} \\
P=p^{-} & \text {for all } & P \in \mathbf{P}^{\searrow, i} \\
X(0)=x^{+}(0) & \text { for all } & X \in \mathbf{X}^{\nearrow, i} \\
X(0)=x^{-}(0) & \text { for all } & X \in \mathbf{X}^{\searrow, i}
\end{array}\right.
$$

- $x_{i}^{-}(t)$ is the solution of the system $\dot{\mathbf{X}}=f\left(\mathbf{X}, \mathbf{P}_{E}, \mathbf{P}_{D}, t\right)$ with the configuration $\mathscr{H}^{i} \in \mathscr{H}$ such that

$$
\underline{\mathscr{H}}^{i}=\left\{\begin{array}{ccc}
P=p^{+} & \text {for all } & P \in \mathbf{P} \searrow, i \\
P=p^{-} & \text {for all } & P \in \mathbf{P}^{\nearrow, i} \\
X(0)=x^{+}(0) & \text { for all } & X \in \mathbf{X}^{\searrow, i} \\
X(0)=x^{-}(0) & \text { for all } & X \in \mathbf{X}^{\nearrow, i}
\end{array}\right.
$$




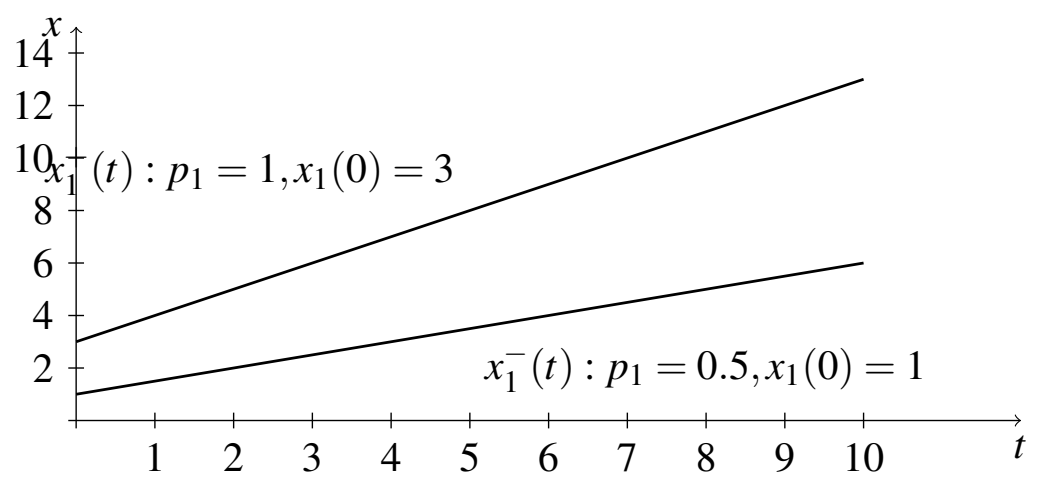

Figure 3: Interval analysis with Example 2 model

This means that if the monotonic properties of $f_{i}$ are known, then one can solve the system with configuration $\overline{\mathscr{H}}^{i}$ to get $x_{i}^{+}(t)$, and with $\underline{\mathscr{H}}^{i}$ to get $x_{i}^{-}(t)$. This means that to retrieve the bounds of $\left[x_{i}\right](t)$ for any variable $X_{i}, i=1, \ldots, m$, the system $\dot{\mathbf{X}}=f\left(\mathbf{X}, \mathbf{P}_{E}, \mathbf{P}_{D}, t\right)$ has to be solved twice with usual techniques not involving intervals. For such systems, propagating uncertainties modelled by intervals then becomes much easier than propagating probabilistic uncertainties (it comes down to solve at most $2 \cdot m$ different systems). Let us continue our example.

Example 2. Consider the system of Example 1. We have (assuming a positive $P_{1}$ ) that $\frac{\partial f_{1}}{\partial P_{1}} \geq 0$, hence $f_{1}$ is dynamically increasing w.r.t. $P_{1}$ and $X_{1}$. Hence $\mathbf{P}^{\nearrow, i}=P_{1}, \mathbf{P}^{\searrow, i}=\emptyset, \mathbf{X}^{\nearrow, i}=X_{1}$ and $\mathbf{X}^{\searrow, i}=\emptyset$. Hence the upper envelope $x_{1}^{+}(t)$ is given by the configuration $\overline{\mathscr{H}}^{1}=\left\{p_{1}^{+}, x^{+}(0)\right\}$, and the lower one by $x_{1}^{-}(t)$ by $\underline{\mathscr{H}^{1}}=\left\{p_{1}^{-}, x^{-}(0)\right\}$. Figure 3 represents the two envelopes when $\left[p_{1}\right]=[0.5,1]$ and $\left[x_{1}\right](0)=[1,3]$ for $t \in[0,10]$.

\subsection{Parameters optimisation}

We now consider the problem of searching optimal values $\widehat{\mathbf{p}}_{D}$ of design parameters $\mathbf{P}_{D}$ when initial conditions and environmental parameters are intervalvalued, and when objectives (constraints) $\widehat{\mathbf{x}}(t)$ on the state variables are intervalvalued as well. We consider that, for each variable $X_{i}$, the objectives $\widehat{x_{i}}: \widehat{\mathscr{T}} \rightarrow \mathbb{I} \mathbb{R}$ can be given over some subset $\widehat{\mathscr{T}} \subseteq \mathscr{T}$ of the whole time domain (for instance, constraints on values may only be specified for the steady state only, that is after a time $t^{*}$ such that $\left.\widehat{\mathscr{T}}=\left[t^{*}, \bar{t}\right], \ldots\right)$. Note that objectives can be intervals, as their exact values can themselves be uncertainly known (or there may be many values 
that appear as optimal in the context). This way of formulating an optimisation problem is not usual, even in interval-analysis literature [22], where the objective function is usually precisely valued (e.g., corresponds to precise outputs). Again, such a problem may be in general difficult to solve. We propose a general way of formulating the problem, before proposing an easy-to-apply algorithm in the case of monotonic systems (i.e., systems satisfying Definition 1).

Consider some pre-defined objectives $\left[\widehat{x}_{i}\right](t)$ as well as some initial conditions $\left[x_{i}\right](0), i=1, \ldots, m$ and some interval-valued uncertainty $[p]$ for every environmental parameter $P \in \mathbf{P}_{E}$. Consider some (precise) value $\widehat{\mathbf{p}_{D}}$ for design parameters $\mathbf{P}_{D}$. Design parameter values $\widehat{\mathbf{p}_{D}}$ are said to form a guaranteed (resp. possible) solution if the solution of the system $\dot{\mathbf{X}}=f\left(\mathbf{X}, \mathbf{P}_{E}, \mathbf{P}_{D}, t\right)$ with these values $\widehat{\mathbf{p}_{D}}$ (and with given intervals on $\mathbf{P}_{E}$ and $\mathbf{X}$ initial conditions) is such that $\left[x_{i}\right](t) \subseteq\left[\widehat{x}_{i}\right](t)$ (resp. $\left[x_{i}\right](t) \cap\left[\widehat{x}_{i}\right](t) \neq \emptyset$ ) for every $t \in \widehat{\mathscr{T}}$. We denote the set of guaranteed solutions by $\mathscr{G}_{D}$ and the set of possible solutions by $\mathscr{S}_{D}$. Both these sets may be empty, but we have the inclusion relationship $\mathscr{G}_{D} \subseteq \mathscr{S}_{D}$. A guaranteed solution is such that, despite of interval uncertainties, we are certain that with the given design parameter values, the true answer lies within the objective bounds, while a possible solution is such that, with the given design parameter values, the true answer may or may not lie within the objective bounds. Solutions that are totally outside the objective bounds are said non-admissible.

Example 3. Consider again the model of Example 2, except that this time parameter $P_{1}$ is considered as a design parameter that can be tuned through some control process (e.g., a speed of reaction controlled by some catalyser). Objective $\widehat{x}_{1}(t)$ on the variable $X_{1}$ is specified for $\widehat{T}=\{10\}$ and is such that $\left[\widehat{x}_{1}\right](10)=[8,11]$. Figure 4 illustrates the notions of guaranteed, possible and non-admissible solutions for this case. For example, when $P_{1}=0.75$, we have $\left[x_{1}\right](10)=\left[x_{1}{ }^{-}, x_{1}{ }^{+}\right](10)=$ $[8.5,10.5]$ and $\left[x_{1}\right](10) \subseteq\left[\widehat{x}_{1}\right](10)$.

The exact bounds of sets $\mathscr{G}_{D}$ and $\mathscr{S}_{D}$ are, in practice, hard to find. However one may search, for each $P \in \mathbf{P}_{D}$, intervals $\left[G_{P}\right]=\left[g_{P}{ }^{-}, g_{P}{ }^{+}\right]$and $\left[S_{P}\right]=\left[s_{P}{ }^{-}, s_{P}{ }^{+}\right]$ approximating $\mathscr{G}_{D}$ and $\mathscr{S}_{D}$ on each design dimension. Those values are the solutions of the following optimisation problems (provided such solutions exist):

$$
g_{P}{ }^{-}=\min P, \quad g_{P}{ }^{+}=\max P,
$$

under the constraints

$$
\begin{gathered}
x_{i}(0) \in\left[x_{i}\right](0), i \in\{1, \ldots, m\}, \quad p_{j} \in\left[p_{j}\right] \forall P_{j} \in \mathbf{P}_{E} \\
\forall t \in \widehat{\mathscr{T}}, i \in\{1, \ldots, m\}, \quad x_{i}^{+}(t) \leq \widehat{x}_{i}(t)^{+} \text {and } x_{i}^{-}(t) \geq \widehat{x}_{i}(t)^{-},
\end{gathered}
$$




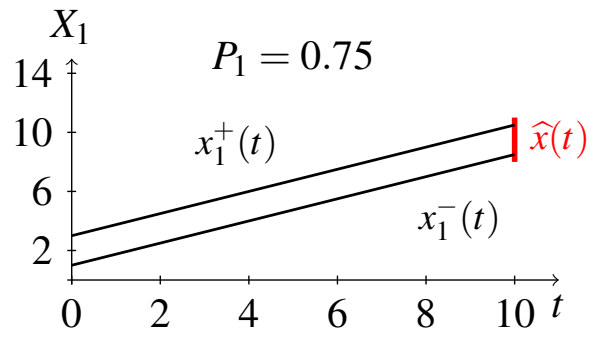

Fig 4.A guaranteed solution

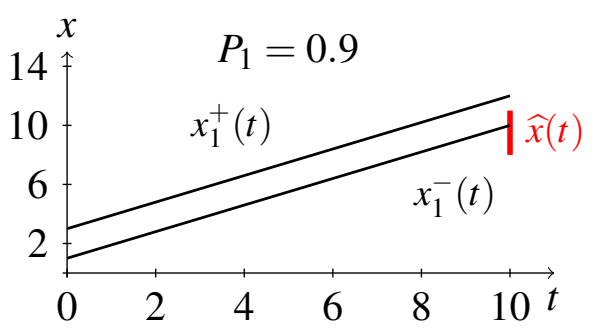

Fig 4.B possible solution

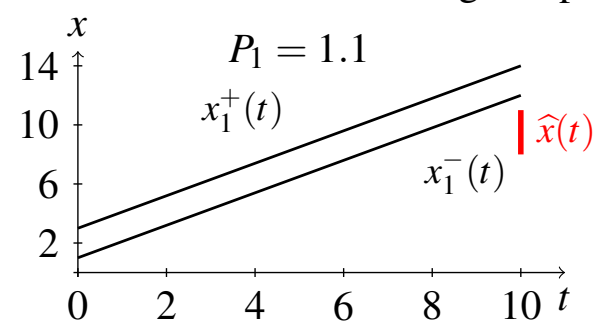

Fig 4.C non-admissible solution

Figure 4: Illustration of solutions with Example 2 model

and

$$
s_{P}{ }^{-}=\min P, \quad s_{P}^{+}=\max P,
$$

under the constraints

$$
\begin{gathered}
x_{i}(0) \in\left[x_{i}\right](0), i \in\{1, \ldots, m\}, \quad p_{j} \in\left[p_{j}\right] \forall P_{j} \in \mathbf{P}_{E} \\
\forall t \in \widehat{\mathscr{T}}, i \in\{1, \ldots, m\}, \quad x_{i}^{-}(t) \leq \widehat{x}_{i}(t)^{+} \text {or } x_{i}^{+}(t) \geq \widehat{x}_{i}(t)^{-} .
\end{gathered}
$$

Again, solving exactly such problems is in general difficult. However, when the $\operatorname{model}^{1} \dot{\mathbf{X}}=f\left(\mathbf{X}, \mathbf{P}_{E}, \mathbf{p}_{D}, t\right)$ is dynamically monotonic (either increasing or decreasing) in each variable and each environmental parameter, we propose a simple heuristic method to identify $\left[G_{P}\right]$ and $\left[S_{P}\right]$. Algorithm 1 suggests some means to find them when each component of $\dot{\mathbf{X}}=f\left(\mathbf{X}, \mathbf{P}_{E}, \mathbf{p}_{D}, t\right)$ is dynamically monotonic w.r.t. a given design parameter $P$. In this algorithm, $\underline{\mathscr{H}}_{\mathbf{P}_{E}, \mathbf{X}}^{i}$ and $\bar{H}_{\mathbf{P}_{E}, \mathbf{X}}^{i}$ are the configurations of Proposition 1 reduced to environmental parameters and initial conditions, the values of design parameters being left unspecified.

The algorithm simply uses the known monotonic properties of the model to compute boundary values. For instance, consider Lines 2-3 and the case $P \in$

\footnotetext{
${ }^{1}$ Note that design parameters $\mathbf{p}_{D}$ are no longer uncertain.
} 


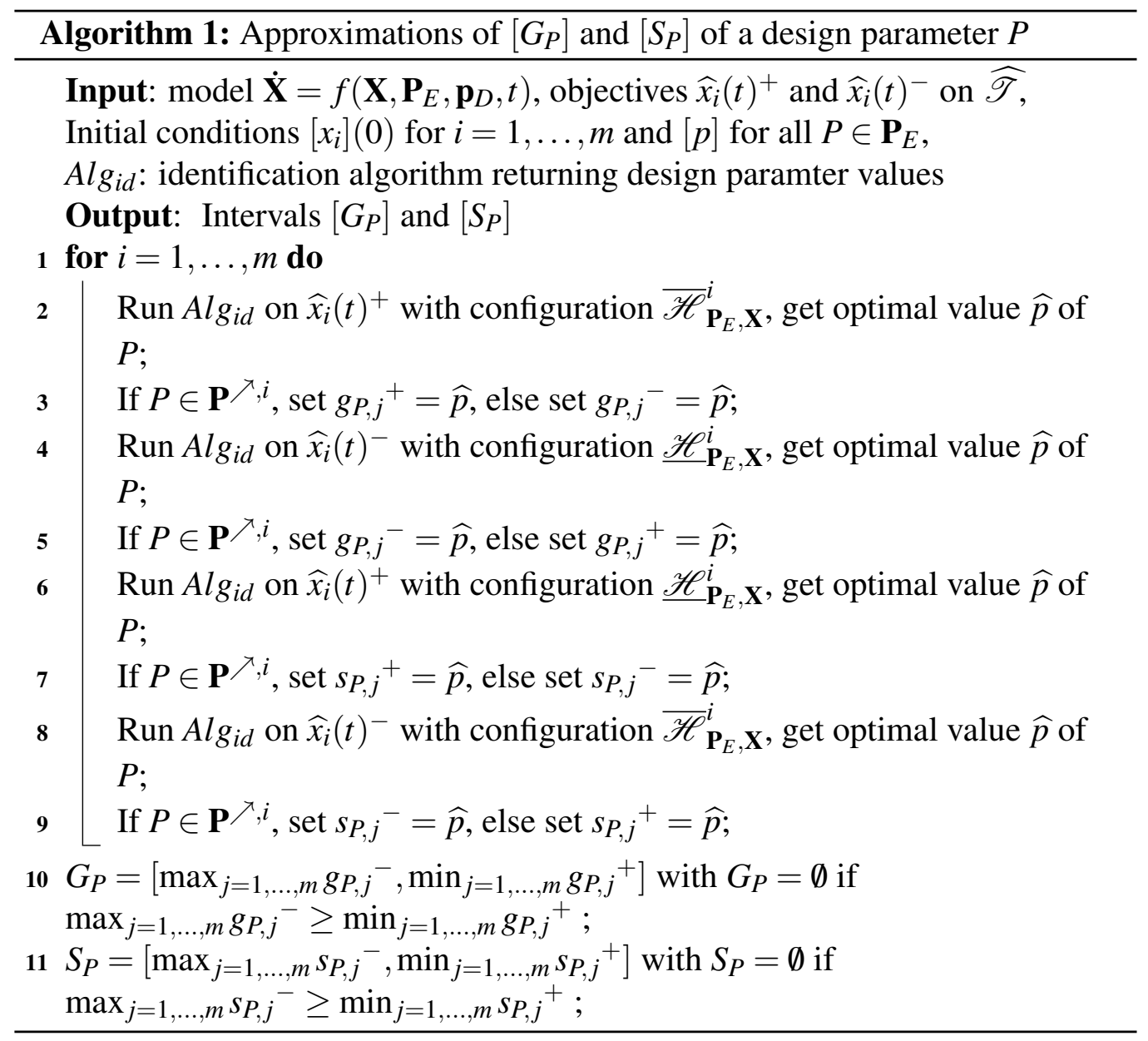


$\mathbf{P}^{\nearrow, i}$, then considering the configuration of environmental parameters and initial conditions giving $x_{i}^{+}(t)$ is the most constraining configuration we can have w.r.t. the objective upper bound $\widehat{x}_{i}(t)^{+}$and such that there is still a chance that $\widehat{p}$ is a guaranteed solution. We still have $\left[S_{P}\right] \subseteq\left[G_{P}\right]$. Note that Algorithm 1 works only when $f$ satisfies Definition 1 . In more complex cases, approximating sets $\mathscr{G}_{D}$ and $\mathscr{S}_{D}$ can be done by sampling different values of design parameters and then performing interval analysis (with propagation methods adapted to more general models [23]) to check whether the sampled values are (guaranteed or possible) solutions.

Example 4. Consider again the model of Example 2, with the constraints of Example 3. Running Algorithm 1 to identify $\left[G_{P_{1}}\right]$ and $\left[S_{P_{1}}\right]$ gives the following solutions (note that here, initial conditions and objectives $\left[\widehat{x}_{1}\right](10)$ provides each time two points, hence totally determining $P_{1}$ in the equation $x_{1}(t)=P_{1} \cdot t+x_{1}(0)$ ):

- Line 2 of Alg. 1, i=1: Identification with $\widehat{x}_{1}^{+}(10)=11$ and $\overline{\mathscr{H}}_{\mathbf{P}_{E}, \mathbf{X}}^{i}=\left\{x_{1}(0)=\right.$ $3\}$, giving $\widehat{p}=0.8$ as solution and $g_{P_{1}, 1}{ }^{+}=0.8$ (since $P_{1} \in \mathbf{P}^{\nearrow, 1}$ ).

- Line 4 of Alg. 1, i=1: Identification with $\widehat{x}_{1}^{-}(10)=8$ and $\underline{\mathscr{H}}_{\mathbf{P}_{E}, \mathbf{X}}^{i}=\left\{x_{1}(0)=\right.$ $1\}$, giving $\widehat{p}=0.7$ as solution and $g_{P_{1}, 1}{ }^{-}=0.7$

- Line 6 of Alg. 1, i=1: Identification with $\widehat{x}_{1}^{+}(10)=11$ and $\underline{H}_{\mathbf{P}_{E}, \mathbf{X}}^{i}=\left\{x_{1}(0)=\right.$ $1\}$, giving $\widehat{p}=1$ as solution and $s_{P_{1}, 1}{ }^{+}=1$

- Line 8 of Alg. 1, i=1: Identification with $\widehat{x}_{1}(10)=8$ and $\overline{\mathscr{H}}_{\mathbf{P}_{E}, \mathbf{X}}^{i}=\left\{x_{1}(0)=\right.$ $3\}$, giving $\widehat{p}=0.5$ as solution and $s_{\mathbf{p}_{1}, 1}{ }^{-}=0.5$

Finally (Lines 10 and 11 of Alg. 1), we obtain intervals $\left[G_{P_{1}}\right]=[0.7,0.8]$ and $\left[S_{P_{1}}\right]=[0.5,1]$ providing approximate values of $P_{1}$ that give guaranteed and possible solutions, respectively.

The obtained sets $\left[S_{P}\right]$ and $\left[G_{P}\right]$ for each design parameter $P \in \mathbf{P}_{D}$ can then be transformed into fuzzy sets [29]. Indeed, while guaranteed solutions all provide the same satisfaction to the designer or the decision maker (they all ensure that the true solution is within the objective boundaries), possible solutions can be seen as having gradual satisfaction degrees, as some of them will have a more significant overlap with the objective bounds than others.

First recall that a fuzzy set $\mu: \mathscr{V} \rightarrow[0,1]$ is a mapping from a space $\mathscr{V}$ (here the real line) to the unit interval, where $\mu(v)$ is the membership degree of element 


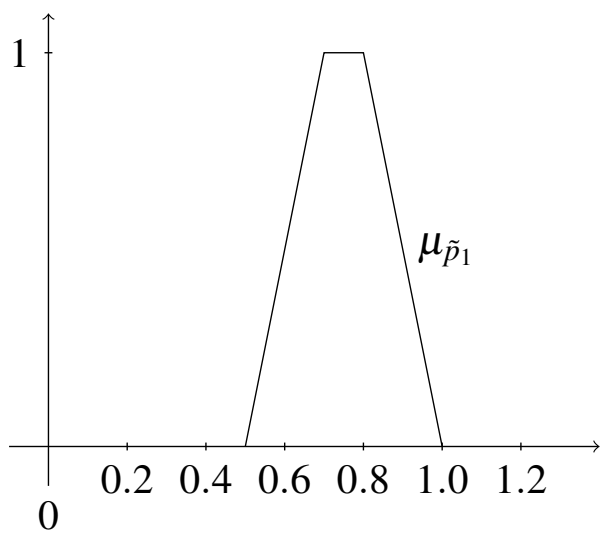

Figure 5: Fuzzy set of Example 4

v. A trapezoidal fuzzy number $\mu_{\tilde{a}}:\left[a_{1}, a_{4}\right] \rightarrow[0,1]$ is defined by a tuple $\tilde{a}=$ $\left\{a_{1}, a_{2}, a_{3}, a_{4}\right\}$ of four numbers and is such that

$$
\mu_{\tilde{a}}(x)= \begin{cases}\frac{x-a_{1}}{a_{2}-a_{1}} & \text { if } a_{1} \leq x \leq a_{2} \\ 1 & \text { if } a_{2} \leq x \leq a_{3} \\ \frac{x-a_{4}}{a_{3}-a_{4}} & \text { if } a_{3} \leq x \leq a_{4}\end{cases}
$$

In our case, the fuzzy set degree expresses some satisfaction degree [8] provided by a parameter value w.r.t. an interval-valued objective. We propose, for a design parameter $P$, to build the trapezoidal fuzzy number $\mu_{\tilde{P}}$ such that $\tilde{P}=$ $\left\{s_{P}{ }^{-}, g_{P}{ }^{-}, g_{P}{ }^{+}, s_{P}{ }^{+}\right\}$if $G_{\mathbf{p}_{i}} \neq \emptyset$ and such that $\tilde{P}=\left\{s_{P}{ }^{-},\left(s_{P}{ }^{-}+s_{P}{ }^{+}\right) / 2,\left(s_{P}{ }^{-}+s_{P}{ }^{+}\right) / 2, s_{P}{ }^{+}\right\}$ otherwise. Figure 4 illustrates the fuzzy number obtained in Example 4.

\subsection{Sensitivity analysis}

When performing an uncertainty analysis, it is usual to perform it along with a sensitivity analysis $[13,25,14]$. Sensitivity analysis consists in searching what input parameters or variables most contribute to the output uncertainty. Its results indicate the parameter or variable on which experimental efforts should concentrate in order to reduce the output uncertainty. Note that there are only very few works dealing with sensitivity analysis of interval approaches [18], contrarily to probabilistic approaches.

In this paper, we propose a very simple method to perform this sensitivity analysis for a given function $f_{i}$. Let $\left[x_{i}\right](t)$ be the interval-valued output resulting from initial interval uncertainty. Then, if we denote by $\left.L\left(x_{i}\left(t_{j}\right)\right):=\left(x_{i}^{(} t_{j}\right)+-x_{i}^{-}\left(t_{j}\right)\right)$ 
the length of $\left[x_{i}\right]\left(t_{j}\right)$ for any time-step $t_{j}$, we can define the overall imprecision $I\left(x_{i}(t)\right)$ of $x_{i}(t)$ as

$$
I\left(x_{i}(t)\right):=\sum_{j=1}^{T} L\left(x_{i}\left(t_{j}\right)\right),
$$

that is, the sum of interval lengths obtained at each time step. Now, to quantify the impact of each parameter and variable uncertainty on the output imprecision, we propose the following procedure (similar to some existing propositions in imprecise probability literature [10]): reduce, for each parameter and variable, its uncertainty by a given fraction $r \in[0,1]$, such a reduction coming down to transform an interval $[a, b]$ into an interval $\left[a^{\prime}, b^{\prime}\right]$ such that

$$
a^{\prime}=M([a, b])-\left(\frac{L([a, b])}{2}(1-r)\right), b^{\prime}=M([a, b])+\left(\frac{L([a, b])}{2}(1-r)\right),
$$

with $M([a, b]), L([a, b])$ the middle and length of the interval, respectively.

Such a reduction gives a new interval-valued solution $\left[x_{i}^{\prime}\right](t)$ included in the previous one, and with an overall imprecision $I\left(x_{i}^{\prime}(t)\right) \leq I\left(x_{i}(t)\right)$. If $\left[x_{i}^{\prime}\right](t)$ is the output obtained after reducing the imprecision of parameter $P$ (or variable $X$ ) by $r$, the gain in the output precision generated by this reduction can then be defined as

$$
G(P, r):=\frac{\left(I\left(x_{i}(t)\right)-I\left(x_{i}^{\prime}(t)\right)\right.}{I\left(x_{i}(t)\right)} .
$$

Let us illustrate this notion in our example.

Example 5. Let us consider again the model of Example 1, with initial uncertainty given by $p_{1} \in[0.5,1]$ and $x_{1}(0) \in[1,3]$, the resulting $x_{1}(t)$ being pictured in Figure 3. We have $I\left(x_{i}(t)\right)=70$ (in this case, the area between the two lines of Figure 3 can be computed analytically).

If we reduce the uncertainty of $P_{1}$ and $X_{1}(0)$ by $50 \%(r=0.5)$, we get $p_{1}^{\prime} \in$ $[0.625,0.875]$ and $x_{1}^{\prime}(0) \in[1.5,2.5]$. We then have

$$
G\left(P_{1}, 0.5\right)=70-45 / 70 \simeq 0.36, G\left(X_{1}, 0.5\right)=70-60 / 70 \simeq 0.14 .
$$

From these results, it appears that reducing the uncertainty of $P_{1}$ by $50 \%$ has a more important impact on the output than reducing $X_{1}(0)$ uncertainty by the same amount. Hence, further experiments should focus on reducing the uncertainty around $P_{1}$.

Finally, note that a reduction of $100 \%(r=1)$ of a parameter or variable comes down to consider the middle point of the interval it belongs to. 


\section{Application to food packaging}

In this section, we apply the above framework to the design and optimisation of food packaging material, with the aim to maximise shelf life of packaged food (here, fresh fruits and vegetables). Note that this model will be integrated to an online decision support system (http://www.tailorpack.com/), hence quick computations and approximate (but reliable) answers should be privileged over more exact but computationally more demanding answers.

\subsection{Problem presentation and model analysis}

Preserving fresh food after harvest and on the shop shelves is an important issue in the food industry. Providing food with optimised packaging is a sure way to avoid premature decay and prolong food edibility. Such optimisation means that the packaging must be permeable enough to oxygen and carbon dioxide so as to allow food respiration, but not too permeable to these two gases, so that maturation and decaying process are slowed down.

In modified atmosphere packaging, oxygen and carbon dioxide partial pressures in packaging head-space are modified and settle to steady values after a transient phase. This modification in the internal gas partial pressures is achieved due to the mass balance between oxygen and carbon dioxide flux through the packaging material and $\mathrm{O} 2$ and $\mathrm{CO} 2$ consumption/production due to the product respiration. For a given fruit or vegetable, it is possible to experimentally determine (up to some uncertainties) oxygen and carbon dioxide partial pressures that will result in an optimal preservation. From these optimal partial pressure values, it is then possible to determine optimal packaging permeances, by using a mathematical model predicting the dynamic evolution of internal gases partial pressures. Several environmental parameters must be specified in this model such as respiration rate or respiratory quotient, packaging geometry, or environment variables such as temperature. The mass balance between gas transfer and respiration can be written as:

$$
\begin{aligned}
p_{\mathrm{O}_{2}}^{\dot{p} k g} & =\frac{P e_{\mathrm{O}_{2}} \cdot S}{e}\left(p_{\mathrm{O}_{2}}^{e x t}-p_{\mathrm{O}_{2}}^{p k g}\right)-R R_{\mathrm{O}_{2}} \cdot m=f_{1} \\
p_{\mathrm{CO}_{2}}^{\dot{p k g}} & =\frac{P e_{\mathrm{CO}_{2}} \cdot S}{e}\left(p_{\mathrm{CO}_{2}}^{e x t}-p_{\mathrm{CO}_{2}}^{p k g}\right)+R R_{\mathrm{O}_{2}} \cdot m \cdot Q R=f_{2}
\end{aligned}
$$




\begin{tabular}{ccc} 
Parameter & Name & Units \\
\hline $\mathrm{Pe}_{\mathrm{O}_{2}}$ & $\mathrm{O}_{2}$ permeance & $\mathrm{mol} \cdot \mathrm{m}^{-1} \cdot \mathrm{s}^{-1} \cdot \mathrm{Pa}^{-1}$ \\
$\mathrm{Pe}_{\mathrm{CO}_{2}}$ & $\mathrm{CO}_{2}$ permeance & $\mathrm{mol} \cdot \mathrm{m}^{-1} \cdot \mathrm{s}^{-1} \cdot \mathrm{Pa}^{-1}$ \\
$\mathrm{~S}$ & pack. surface & $\mathrm{m}^{2}$ \\
$e$ & pack. thickness & $\mathrm{m}$ \\
$p_{\mathrm{j}}^{i}$ & partial press. of $\mathrm{j}$ in i & $\%$ \\
$R R_{\mathrm{O}_{2}}$ & $\mathrm{O}_{2}$ respiratory rate & $\mathrm{mmol} \cdot \mathrm{kg}^{-1} \cdot \mathrm{h}^{-1}$ \\
$\mathrm{RR}_{\mathrm{O}_{2} \mathrm{max}}$ & max. $\mathrm{O}_{2}$ respiratory rate & $\mathrm{mmol} \cdot \mathrm{kg}^{-1} \cdot \mathrm{h}^{-1}$ \\
$\mathrm{Km}_{\mathrm{appO}}$ & Michaëlis-Menten constant & $\mathrm{kPa}$ \\
$\mathrm{Ki}_{\mathrm{CO}_{2}}$ & $\mathrm{CO}_{2}$ inhibition constant & $\mathrm{kPa}$ \\
$m$ & food mass & $\mathrm{kg}$ \\
$\mathrm{QR}$ & respiration coefficient & $\times$
\end{tabular}

Table 1: Parameters names and units. $\times=$ non-relevant.

with

$$
R R_{O_{2}}=\frac{R R_{O_{2} \max } \cdot p_{O_{2}}^{p k g}}{\left(K m_{a p p O_{2}}+p_{O_{2}}^{p k g}\right) \cdot\left(1+\frac{p_{C O_{2}}^{p k g}}{K i_{C O_{2}}}\right)}
$$

where the first part of the right-hand side describes gas flux per time unit through the packaging material, while the second part describes gas consumption (and emission) by the vegetable or fruit (modelled using a Michaëlis-Menten-type equation, see (13)). There are two variables $\mathbf{X}=\left\{p_{\mathrm{O}_{2}}^{p k g}, p_{\mathrm{CO}_{2}}^{p k g}\right\}$. Table 1 summarises the parameters with their names and units, while Table 2 summarises whether they are variable, environmental or design parameters, and their monotonic w.r.t. each function $f_{1}$ and $f_{2}$. Note that $\left(p_{\mathrm{O}_{2}}^{e x t}-p_{\mathrm{O}_{2}}^{p k g}\right)$ is always positive, while $\left(p_{\mathrm{CO}_{2}}^{e x t}-p_{\mathrm{CO}_{2}}^{p k g}\right)$ is always negative, that $p_{i}^{e x t}$ are constant and that all parameters are positive (otherwise model would not be monotonic w.r.t. every parameter).

\subsection{Case study: chicory}

Note that in our case study, the shape of the packaging ( $S$ and $e$ ) and the mass $(m)$ of food inside it have already been fixed, so that the only parameters that can be adjusted to optimise the packaging are the material permeability properties $\left(\mathrm{Pe}_{\mathrm{O}_{2}}\right.$ and $\left.\mathrm{Pe}_{\mathrm{CO}_{2}}\right)$. Note that, if a probabilistic approach was used, no less than 9 probability distributions would have to be specified, and independence between variables would have to be assumed, with some of the parameters sometimes measured only 3 times. Also, estimating boundaries by interval analysis for $p_{\mathrm{O}_{2}}^{p k g}$ and 


\begin{tabular}{|c|c|c|c|}
\hline Parameter & $\mathbf{P}_{D}, \mathbf{P}_{E}, \mathbf{X}$ & $f_{1}$ & $f_{2}$ \\
\hline $\mathrm{Pe}_{\mathrm{O}_{2}}$ & $\in \mathbf{P}_{D}$ & $\mathbf{P}^{\nearrow}, 1$ & $x$ \\
\hline $\mathrm{Pe}_{\mathrm{CO}_{2}}$ & $\in \mathbf{P}_{D}$ & $\times$ & $\mathbf{P} \searrow, 2$ \\
\hline$S$ & $\in \mathbf{P}_{E}$ & $\mathbf{P}^{\nearrow, 1}$ & $\mathbf{P} \searrow, 2$ \\
\hline$e$ & $\in \mathbf{P}_{E}$ & $\mathbf{P} \searrow, 1$ & $\mathbf{P}^{\nearrow, 2}$ \\
\hline$p_{j}^{i}$ & $\in \mathbf{X}$ & $\mathbf{X} \searrow, 1$ & $\mathbf{X} \searrow, 2$ \\
\hline $\mathrm{RR}_{\mathrm{O}_{2}}$ & $\in \mathbf{P}_{E}$ & $\mathbf{P} \searrow, 1$ & $\mathbf{P}^{\nearrow, 2}$ \\
\hline$R R_{O_{2} \max }$ & $\in \mathbf{P}_{E}$ & $\mathbf{P} \searrow, 1$ & $\mathbf{P}^{\nearrow, 2}$ \\
\hline$K m_{\text {appO }_{2}}$ & $\in \mathbf{P}_{E}$ & $\mathbf{P}^{\nearrow, 1}$ & $\mathbf{P} \searrow, 2$ \\
\hline$K i_{\mathrm{CO}_{2}}$ & $\in \mathbf{P}_{E}$ & $\mathbf{P} \searrow, 1$ & $\mathbf{P}^{\nearrow, 2}$ \\
\hline$m$ & $\in \mathbf{P}_{E}$ & $\mathbf{P} \searrow, 1$ & $\mathbf{P}^{\nearrow, 2}$ \\
\hline$Q R$ & $\in \mathbf{P}_{E}$ & $\times$ & $\mathbf{P}^{\nearrow, 2}$ \\
\hline
\end{tabular}

Table 2: Parameters type and monotonic w.r.t. $f_{1}, f_{2} . \times=$ non-relevant.

$p_{\mathrm{CO}_{2}}^{p k g}$ here requires only 4 simulations (far less than for a Monte-Carlo simulation [13]).

The considered food is chicory, which has been previously studied, but without any proper uncertainty analysis [5].

\subsubsection{Uncertainty propagation step}

Knowing the permeability of the packaging material classically used to pack chicory, the mathematical model described by Eq (11)-(13) can be used to simulate the evolution with time of the internal $\mathrm{O} 2$ and $\mathrm{CO} 2$ partial pressures. Parameters with their interval uncertainty are summarised in Table 3. Maximal shelf life is estimated to be 200 hours, therefore the simulation time domain is $\mathscr{T}=[0,200] \mathrm{h}$ and the domain is discretised so that each time step is one minute.

The results of interval analysis are displayed in Figure 6, and we can see that, at the steady states, $p_{\mathrm{O}_{2}}^{p k g} \in[1.6,8.1] \%$ and $p_{\mathrm{CO}_{2}}^{p k g} \in[1.9,5.3] \%$. Using Proposition 1, only four simulations were needed (two for each gas) to estimate the bounds.

Also note that, in the current case, initial conditions $p_{\mathrm{O}_{2}}^{p k g}(0)$ and $p_{\mathrm{CO}_{2}}^{p k g}(0)$ for the two variables are perfectly known and only parameters are uncertain, as initial partial pressures are the same as in free atmosphere (21\% for oxygen and $0 \%$ for carbon dioxide). Therefore, only uncertainty pertaining to environmental and design parameters $\mathbf{P}_{E}$ and $\mathbf{P}_{D}$ have to be taken into account here (contrary to Example 1, where $x_{1}(0)$ value is also uncertain). 


\begin{tabular}{ccc} 
Parameter & Uncertainty & Unit \\
\hline $\mathrm{Pe}_{\mathrm{O}_{2}}$ & {$[878,1278] 10^{-18}$} & $\mathrm{~mol} . \mathrm{m}^{-1} \cdot \mathrm{s}^{-1} \cdot \mathrm{Pa}^{-1}$ \\
$\mathrm{Pe}_{\mathrm{CO}_{2}}$ & {$[3614,4634] 10^{-18}$} & $\mathrm{~mol} . \mathrm{m}^{-1} \cdot \mathrm{s}^{-1} \cdot \mathrm{Pa}^{-1}$ \\
$\mathrm{~S}$ & {$[12,16] 10^{-2}$} & $\mathrm{~m}^{2}$ \\
$e$ & {$[4,6] 10^{-5}$} & $\mathrm{~m}$ \\
$m$ & {$[0.45,0.55]$} & $\mathrm{kg}$ \\
$\mathrm{RR}_{\mathrm{O}_{2} \max }$ & {$[1.3,1.5]$} & $\mathrm{mmol} \cdot \mathrm{kg}^{-1} \cdot \mathrm{h}^{-1}$ \\
$\mathrm{Km}_{\mathrm{appO}}$ & {$[8.26,10.26] 10^{3}$} & $\mathrm{kPa}$ \\
$\mathrm{Ki}_{\mathrm{CO}_{2}}$ & {$[1025,2025] 10^{3}$} & $\mathrm{kPa}$ \\
$\mathrm{QR}$ & {$[0.67,0.81]$} &
\end{tabular}

Table 3: Parameters uncertainty for propagation.

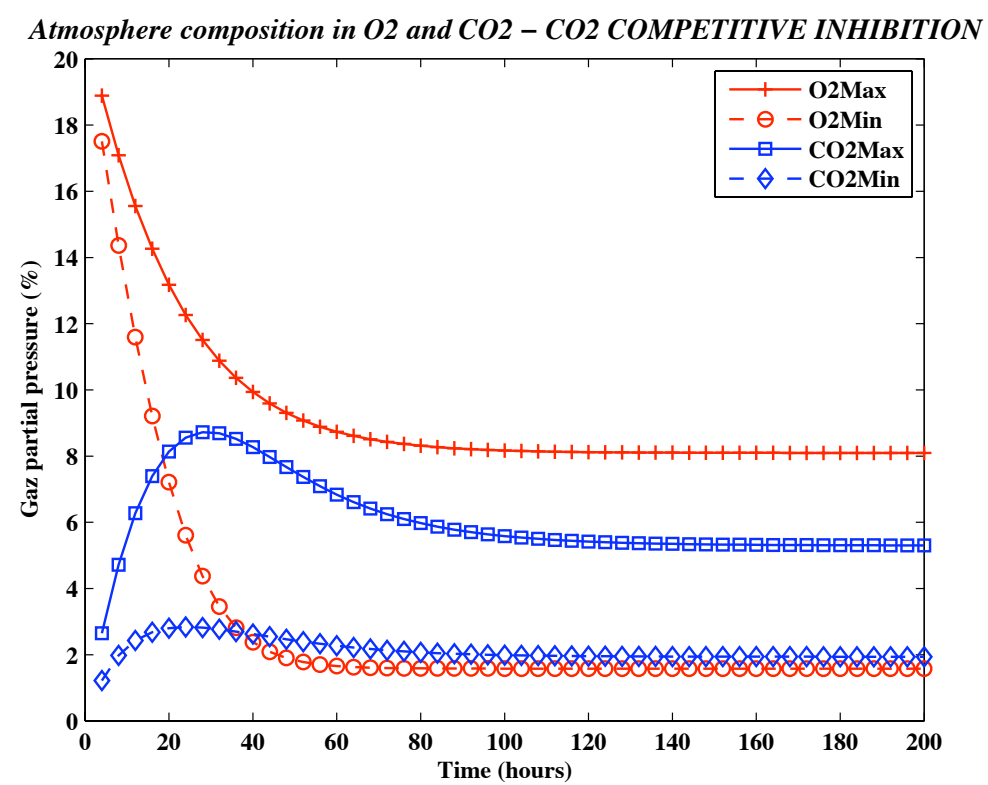

Figure 6: Uncertainty propagation. 


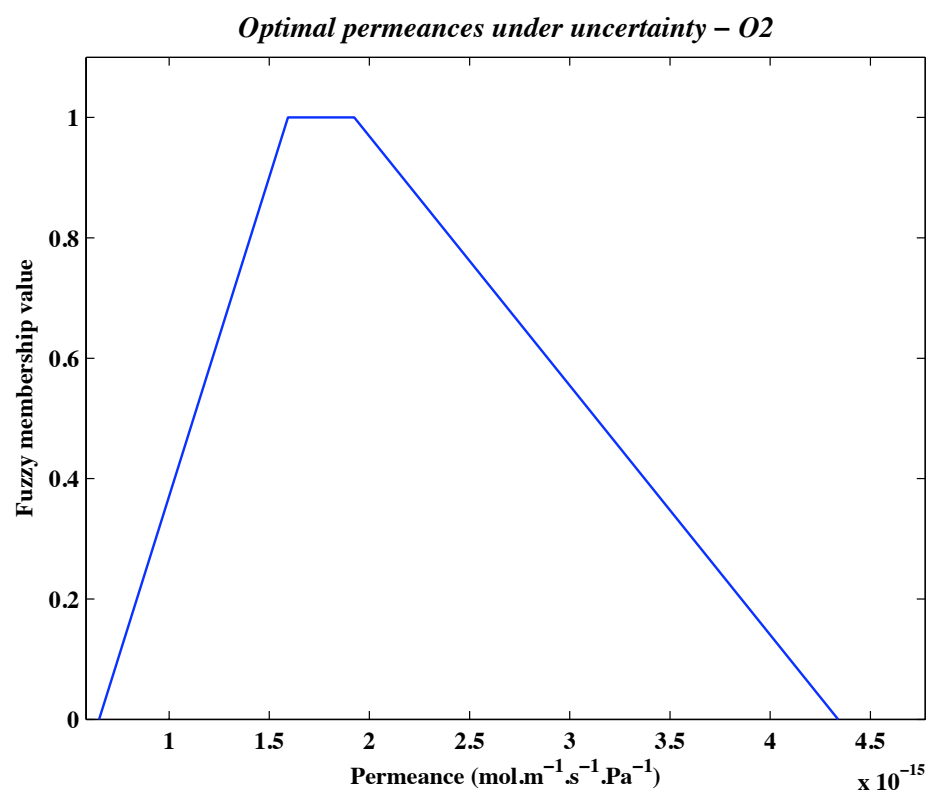

Figure 7: Optimal O2 permeance

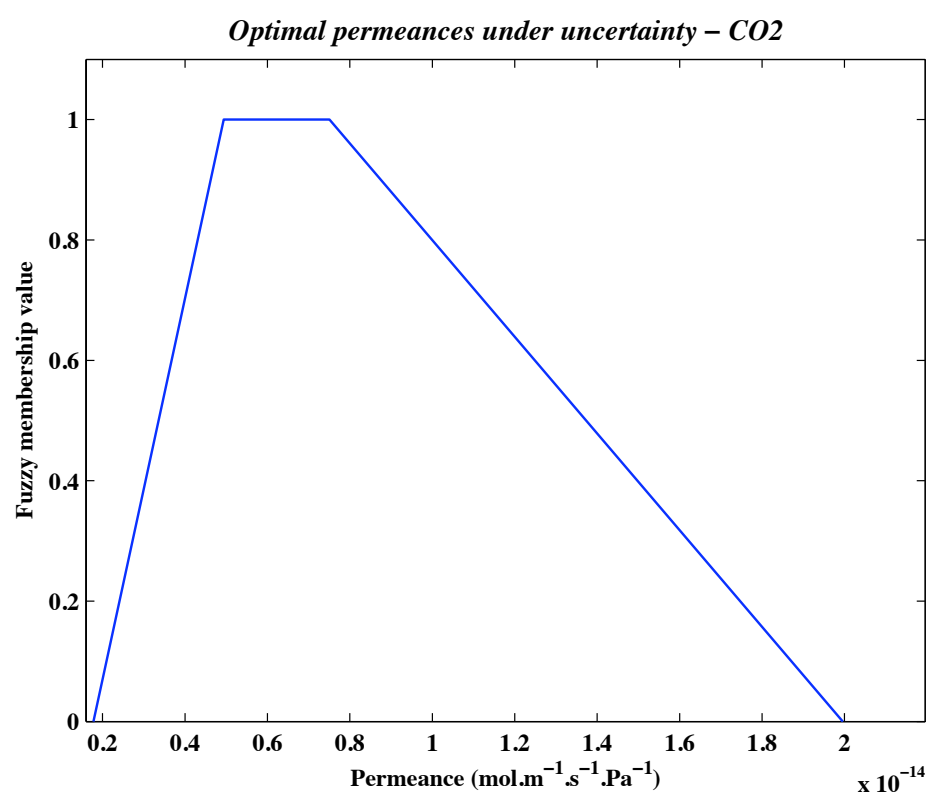

Figure 8: Optimal $\mathrm{CO} 2$ permeance 


\subsubsection{Optimisation step}

In the case where best oxygen and carbon dioxide permeabilities suiting a particular fruit or vegetable are not known a priori, it is possible to use Equations (11)-(13) to perform a reverse engineering task. In this case, optimal oxygen and carbon dioxide concentrations in the packaging modified atmosphere have to be specified. Once this is done, Algorithm 1 can be run to find optimal permeabilities that allow reaching the specified goal.

In this study, Algorithm 1 was run with the same uncertainty on environmental parameters as the one of Table 3 (i.e., all parameters except packaging material permeabilities to $\mathrm{O}_{2}$ and $\mathrm{CO}_{2}$, which are the design parameters). Given that $\mathscr{T}=[0,200] h$ and the fact that there exists a transient phase, we have chosen $\widehat{\mathscr{T}}=[150,200], \widehat{p_{\mathrm{O}_{2}}^{p k g}}(t)=[4,10] \%$ and $\widehat{p_{C O_{2}}^{p k g}}(t)=[2,5] \%$ for every $t \in \widehat{\mathscr{T}}$, that is we want oxygen partial pressure to be between 4 and $10 \%$ and carbon dioxide partial pressure to be between 2 and $5 \%$ at the steady state. Fuzzy sets obtained from Algorithm 1 and from the intervals $\left[G_{P_{O_{2}}}\right]=[1.59,1.92] 10^{-15},\left[G_{P_{C_{C O}}}\right]=$ $[4.94,7.50] 10^{-15}$ and $\left[S_{P_{O_{2}}}\right]=[4.34,6.51] 10^{-15},\left[S_{P_{C_{2}}}\right]=[1.78,19.96] 10^{-15}$ (values are expressed in $\mathrm{mol} . \mathrm{m}^{-1} . \mathrm{s}^{-1} . \mathrm{Pa}^{-1}$ ) are shown in Figures 7 and 8 (LevenbergMarquardt Algorithm [21] was used to identify parameters). Figure 9 shows the result of the interval analysis done with optimal parameters belonging to $\left[G_{P_{O_{2}}}\right] \times\left[G_{P_{C} \mathrm{CO}_{2}}\right]$. We see that the resulting imprecise oxygen and carbon dioxide partial pressures well lie in the objective bounds at the steady state.

\subsection{3. sensitivity analysis}

Table 4 summarises the sensitivity analysis performed according to the method presented in Section 3.4. For each variable $p_{\mathrm{O}_{2}}^{p k g}(t)$ and $p_{\mathrm{CO}_{2}}^{p k g}(t)$, we have evaluated the precision gain after an uncertainty reduction of $r=0.5$ of each (environmental and design) parameter. The last column ( $A l l)$ indicates the gain in the output precision when all parameters uncertainty is reduced by $r=0.5$.

The results indicate, among other things, that while $\mathrm{O}_{2}$ permeability uncertainty has an important impact on both $\mathrm{O}_{2}$ and $\mathrm{CO}_{2}$ internal partial pressures uncertainty (the reduction resulting in a gain of about 0.1 for each), $\mathrm{CO}_{2}$ permeability uncertainty only impact the $\mathrm{CO}_{2}$ internal pressures, and have almost none effects on $\mathrm{O}_{2}$ internal pressure. As could be expected, the respiration rate $Q R$ uncertainty only impact on the $\mathrm{CO}_{2}$ internal pressure, while $\mathrm{Ki}_{\mathrm{CO}_{2}}$ uncertainty, due to the large value of $\mathrm{Ki}_{\mathrm{CO}_{2}}$, has (almost) no impact on the resulting uncertainty for both variables.

Finally, to reduce the most efficiently the uncertainty on $\mathrm{O}_{2}$ and $\mathrm{CO}_{2}$ internal 


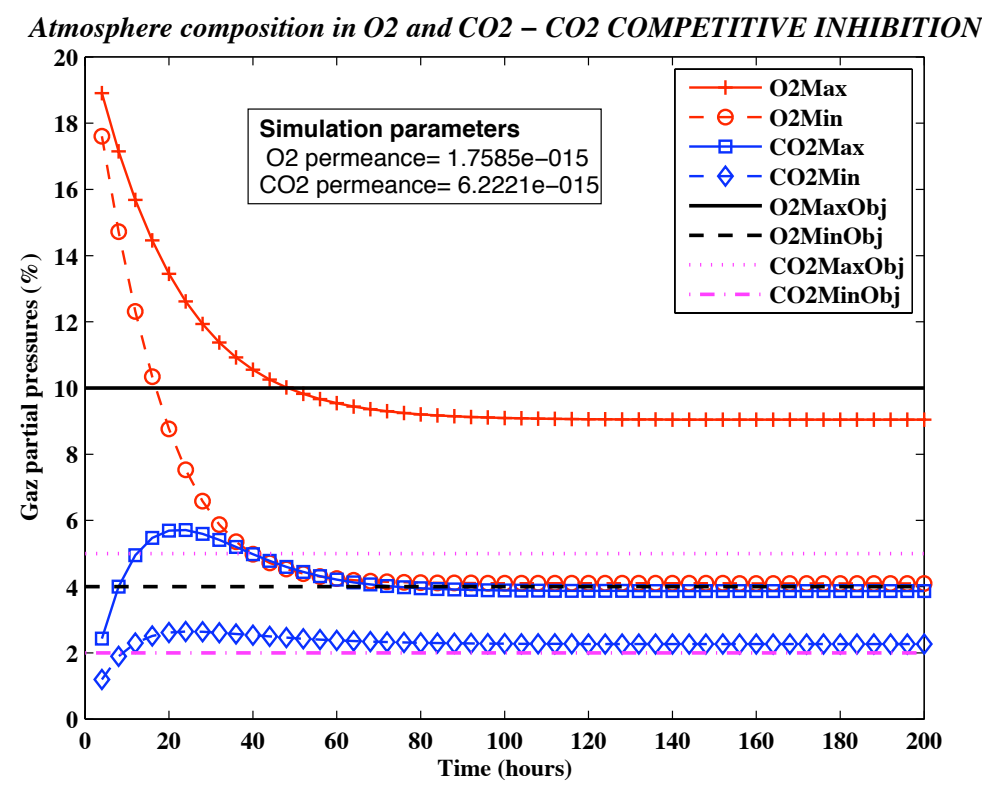

Figure 9: Uncertainty propagation with optimal permeances

\begin{tabular}{c|ccccc}
$P$ & $\mathrm{Pe}_{\mathrm{O}_{2}}$ & $\mathrm{Pe}_{\mathrm{CO}_{2}}$ & $e$ & $S$ & $m$ \\
\hline$p_{\mathrm{O}_{2}}^{p k g}(t)$ & 0.099 & 0 & 0.124 & 0.077 & 0.088 \\
$p_{\mathrm{CO}}^{p k g}(t)$ & 0.095 & 0.12 & 0.077 & 0.058 & 0.024 \\
$P$ & $Q R$ & $R_{O_{2} \max }$ & $\mathrm{Km}_{a p p O_{2}}$ & $\mathrm{Ki}_{\mathrm{CO}_{2}}$ & $\mathrm{All}$ \\
\hline$p_{\mathrm{O}_{2}}^{p k g}(t)$ & 0 & 0.057 & 0.045 & 0 & 0.501 \\
$p_{\mathrm{CO}_{2}}^{p k g}(t)$ & 0.094 & 0.02 & 0.019 & 0 & 0.505
\end{tabular}

Table 4: Values $G(P, 0.5)$ of sensitivity analysis on the parameters $P$ after a reduction $r=0.5$, for the two variables $p_{O_{2}}^{p k g}(t)$ and $p_{C O_{2}}^{p k g}(t)$ 
partial pressures, one should focus on a better characterisation of $\mathrm{O}_{2}$ permeability and thickness, and on $\mathrm{O}_{2} / \mathrm{CO}_{2}$ permeability, respectively.

\section{Conclusion}

Interval analysis is a useful alternative to probabilistic analysis that requires far less information to be applicable, thus avoiding the need to introduce hypothesis unsupported by available information. In some situations where the models satisfy some monotonic properties, using intervals is also more computationally efficient, as sampling methods are not needed to achieve computations. In this paper, we have used interval analysis for two purposes: classical uncertainty propagation for monotonic dynamical models and robust design under uncertainties. We have also proposed an easy method to perform some first sensitivity analysis.

The proposed robust design optimisation method, although approximate, quickly produces optimal values for design parameters. It takes account of interval uncertainty and can cope with imprecisely specified goals, distinguishing between possible solutions (potentially satisfying the goal) and guaranteed solutions (certainly satisfying the goal). The difference between the two kinds of solutions is represented by the means of fuzzy sets describing optimal solutions.

Methodological perspectives to this work include the development of more precise methods, eventually ending up with better and multi-dimensional approximations of the sets $\mathscr{G}_{D}$ and $\mathscr{S}_{D}$. Also, it may be desirable to extend the current approach to hybrid uncertainty models mixing interval and probabilistic uncertainty $[3,2,12,20]$, as information concerning different parameters may vary in quantity and quality. However, these two perspectives would involve more computationally demanding procedures, thus reducing the number of models one could work with.

More applied perspectives include the combination of the optimisation system with a decision support system where a user can search a database for optimal packaging [7] (fuzzy sets describing sets of optimal solutions will then be used as user preferences) and the application of the system to other food products (such as mushrooms).

\section{References}

[1] Aster, R. C., Borchers, B., Thurber, C. H., 2005. Parameter Estimation and Inverse Problems. Academic Press. 
[2] Baudrit, C., Guyonnet, D., Dubois, D., 2006. Joint propagation and exploitation of probabilistic and possibilistic information in risk assessment. IEEE Trans. Fuzzy Systems 14, 593-608.

[3] Baudrit, C., Hélias, A., Perrot, N., 2009. Joint treatment of imprecision and variability in food engineering: Application to cheese mass loss during ripening. Journal of Food Engineering 93 (3), 284-292.

[4] Bedford, T., Cooke, R., 2001. Probabilistic Risk Analysis. Foundations and Methods. Cambridge University Press, UK.

[5] Charles, F., Sanchez, J., Gontard, N., 2005. Modeling of active modified atmosphere packaging of endives exposed to several postharvest temperatures. Journal of Food Science 70, 443-449.

[6] Delanoue, N., 2009. A new method for integrating ode based on monotonicity. In: SWIM 09, A small workshop on Interval Methods.

[7] Destercke, S., Buche, P., Guillard, V., 2011. A flexible bipolar querying approach with imprecise data and guaranteed results. Fuzzy Sets and Systems 169 (1), 51-64.

[8] Dubois, D., Prade, H., 1997. The three semantics of fuzzy sets. Fuzzy Sets Syst. 90 (2), 141-150.

[9] Ferson, S., Ginzburg, L. R., 1996. Different methods are needed to propagate ignorance and variability. Reliability Engineering and System Safety $54,133-144$.

[10] Ferson, S., Tucker, W. T., 2006. Sensitivity analysis using probability bounding. Reliability Engineering and System Safety 91, 1435-1442.

[11] Fortin, J., Dubois, D., Fargier, H., 2008. Gradual numbers and their application to fuzzy interval analysis. IEEE Transactions on Fuzzy Systems 16, $388-402$.

[12] Fuchs, M., Neumaier, A., 2009. Potential based clouds in robust design optimization. Journal of statistical theory and practice 3 (225-238).

[13] Helton, J. C., Johnson, J. D., Oberkampf, W. L., Sallaberry, C. J., 2006. Sensitivity analysis in conjunction with evidence theory representations of 
epistemic uncertainty. Reliability Engineering and System Safety 91, 14141434.

[14] Helton, J. C., Johnson, J. D., Sallaberry, C. J., Storlie, C. B., 2006. Survey of sampling-based methods for uncertainty and sensitivity analysis. Reliability Engineering System Safety 91 (10-11), 1175-1209.

[15] Hertog, M. L., Lammertyn, J., Scheerlinck, N., Nicolki, B. M., 2007. The impact of biological variation on postharvest behaviour: The case of dynamic temperature conditions. Postharvest Biology and Technology 43 (2), $183-192$.

[16] Jaulin, L., Kieffer, M., Didrit, O., Walter, E., 2001. Applied Interval Analysis. London.

[17] Jaulin, L., Kieffer, M., Walter, E., Meizel, D., 2002. Guaranteed robust nonlinear estimation with application to robot localization. IEEE Trans. on Syst., Man and Cybern. C 32 (4), 374-381.

[18] Moens, D., Vandepitte, D., 2007. Interval sensitivity theory and its application to frequency response envelope analysis of uncertain structures. Computer Methods in Applied Mechanics and Engineering 196 (21-24), 2486 2496.

[19] Moore, R., 1979. Methods and applications of Interval Analysis. SIAM Studies in Applied Mathematics. SIAM, Philadelphia.

[20] Nassreddine, G., Abdallah, F., Denoeux, T., 2010. State estimation using interval analysis and belief-function theory: Application to dynamic vehicle localization. IEEE Trans. on Syst., Man and Cybern. B 40, 1205-1218.

[21] Nocedal, J., Wright, S., 1999. Numerical Optimization. Springer, New York.

[22] Raissi, T., Ramdani, N., Candau, Y., 2004. Set membership state and parameter estimation for systems described by nonlinear differential equations. Automatica 40 (10), 1771 - 1777.

[23] Ramdani, N., Meslem, N., Candau, Y., 2009. A hybrid bounding method for computing an over-approximation for the reachable set of uncertain nonlinear systems. IEEE Trans. on Automatic Control 54 (10), 2352-2364. 
[24] Ramdani, N., Meslem, N., Candau, Y., 2010. Computing reachable sets for uncertain nonlinear monotone systems. Nonlinear Analysis: Hybrid Systems 4 (2), $263-278$.

[25] Saltelli, A., Ratto, M., Andres, T., Campolongo, F., Cariboni, J., Gatelli, D., Saisana, M., Tarantola, S., Jan. 2008. Global Sensitivity Analysis: The Primer. WileyBlackwell.

[26] Sandri, S., Dubois, D., Kalfsbeek, H., August 1995. Elicitation, assessment and pooling of expert judgments using possibility theory. IEEE Trans. on Fuzzy Systems 3 (3), 313-335.

[27] Singer, A. B., Barton, P. I., 2006. Bounding the solutions of parameter dependent nonlinear ordinary differential equations. SIAM J. Sci. Comput. 27 (6), 2167-2182.

[28] Walley, P., 1991. Statistical reasoning with imprecise Probabilities. Chapman and Hall, New York.

[29] Zadeh, L., 1975. The concept of a linguistic variable and its application to approximate reasoning-i. Information Sciences 8, 199-249. 